\title{
CAPÍTULO XII \\ GAMING UNA ESTRATEGIA PARA LA REHABILITACIÓN DE PERSONAS CON DISCAPACIDAD
}

\section{Luis Guillermo Molero Suarez}

Doctor en Ciencias Gerenciales. Compensar Unipanamericana Fundación Universitaria Panamericana. Grupo de Investigación en Ingeniería de Sistemas - GIIS. lmolero@ unipanamericana.edu.co ORCID: 0000-0002-4117-8273 Avenida (calle) 32 No. 17-13, Teusaquillo, Bogotá, Colombia.

\section{Pablo Emilio Ospina Rodríguez}

Magister en Ingeniería Electrónica Compensar Unipanamericana Fundación Universitaria Panamericana. Grupo de Investigación en Ingeniería de Sistemas - GIIS. peospinar@ unipanamericana.edu.co Avenida (calle) 32 No. 17-13, Teusaquillo, Bogotá, Colombia.

\section{Martha Liliana Quevedo}

Magister en Software Libre Corporación Universitaria Minuto de Dios - UNIMINUTO mquevedo@uniminuto.edu

\section{José Luis Montenegro Romero}

Tecnólogo en Análisis y Desarrollo de Sistemas de Información. Compensar Unipanamericana Fundación Universitaria Panamericana. Grupo de Investigación en Ingeniería de Sistemas - GIIS. jlmontenegro@unipanamericana.edu.co Avenida (calle) 32 No. 17-13, Teusaquillo, Bogotá, Colombia.o

\section{Luis Daniel Castiblanco Rosero}

Tecnólogo en Análisis y Desarrollo de Sistemas de Información. Compensar Unipanamericana Fundación Universitaria Panamericana. Grupo de Investigación en Ingeniería de Sistemas - GIIS. ldanielcastiblanco@unipanamericana.edu.co Avenida (calle) 32 No. 17-13, Teusaquillo, Bogotá, Colombia.

\section{Resumen:}

El objetivo del estudio es analizar el GAMING desde la perspectiva de un conjunto sistemático de técnicas de desarrollo de aplicaciones y accesorios tecnológicos orientados hacia los videojuegos, como una estrategia para la rehabilitación de personas con discapacidad, haciendo las reflexiones necesarias acerca de la esencia de lo que esta nueva generación de productos tecnológicos permite lograr en estos escenarios. Metodológicamente está orientada bajo el paradigma cualitativo de carácter documental, transaccional, con un diseño no experimental. En este orden de ideas, se desnuda la discapacidad desde la figura de la gamificación y hasta qué punto esta tecnología permite mejorar significativamente o incluso superar diversas discapacidades siendo vista desde diferentes entornos virtuales, los cuales, establecen criterios robustos y probados científicamente para establecer mejorías en cuanto a la rehabilitación se refiere. En el contenido se analizó a detalle los siguientes puntos: 1) GAMING, una filosofía estratégica para casos complejos, 2) Rehabilitación y GAMING, 3) Entornos de Desarrollo para el GAMING en personas con discapacidad. La sistematización de los ciclos de rehabilitación haciendo uso de los ambientes gamificados, 
se han convertido en poderosas herramientas para optimizar estas áreas importantes de la salud, logrando favorecer considerablemente a todo paciente con discapacidad reducida, en contraste, con aquellos pacientes que carecen de ella. Como conclusión, se obtuvo que una ventaja considerable de estos ambientes virtuales gamificados es que pueden recrear diversos escenarios de acuerdo al paciente, estableciendo de esta forma, pautas importantes de las cuales carece el método tradicional. En la actualidad, existen una nutrida gama de entornos para el desarrollo de ambientes gamificados, así como también, herramientas que permiten crear entornos en 3D, realidad virtual o realidad aumentada lo que aumenta la estimulación de los sentidos y refuerza las estrategias para lograr una rehabilitación temprana.

Palabras clave: GAMING, Discapacidad, rehabilitación motriz, Entornos de desarrollo

\title{
GAMING, A STRATEGY FOR THE REHABILITATION OF PERSONS WITH DISABILITIES
}

\begin{abstract}
:
The objective of the study is to analyze the GAMING from the perspective of a systematic set of techniques for the development of applications and technological accessories oriented towards videogames, as a strategy for the rehabilitation of people with disabilities, making the necessary reflections about the essence of the that this new generation of technological products allows to achieve in these scenarios. Methodologically, it is oriented under the qualitative paradigm of a documentary, transactional nature, with a non-experimental design. In this order of ideas, disability is nude from the figure of gamification and to what extent this technology allows significantly improve or even overcome various disabilities being viewed from different virtual environments, which establish robust and scientifically proven criteria to establish improvements in As far as rehabilitation is concerned. In the content, the following points were analyzed in detail: 1) GAMING, a strategic philosophy for complex cases, 2) Rehabilitation and GAMING, 3) Development Environments for GAMING in people with disabilities. The systematization of the rehabilitation cycles using gamified environments, have become powerful tools to optimize these important areas of health, achieving considerably benefit to all patients with reduced disability, in contrast, with those patients who lack it. In conclusion, it was found that a considerable advantage of these virtual gamified environments is that they can recreate various scenarios according to the patient, establishing in this way, important guidelines of which the traditional method lacks. Currently, there are a wide range of environments for the development of gamified environments, as well as tools that allow creating environments in $3 \mathrm{D}$, virtual reality or augmented reality which increases the stimulation of the senses and reinforces the strategies to achieve a rehabilitation early
\end{abstract}

Keywords: GAMING, Disability, motor rehabilitation, Development environments

\section{Capítulo es resultado de un proyecto de investigación intitulado}

"Desarrollo de Suite Interactiva con fines de rehabilitación para personas con movilidad reducida" que se encuentra ya finalizado. Institución financiadora: Unipanamericana Fundación Universitaria Panamericana bajo el Código del proyecto SIGP/Código entidad financiadora número PI242019. 


\section{Introducción}

En la última década, se ha reflejado un incremento considerable en el uso de nuevas tecnologías que han permeado el quehacer de muchas disciplinas específicas, lo que ha traído consigo atrevidos procesos de reingeniería para optimizar estas labores cotidianas a través de equipos y técnicas de última generación, tal es el caso, de las terapias de rehabilitación.

En tal sentido, un términos muy utilizado a la hora de reingeniar estos procesos de rehabilitación y fisioterapia de forma motivadora, inclusiva y novedosa es el GAMING; una novedosa estrategia cuyo término acuña a un grupo de aplicaciones desarrolladas en 3D, Realidad Virtual (RV) o Realidad Aumentada (RA) y accesorios como el Kinect de Microsoft y gafas 3D que permiten a través de videojuegos, juegos serios o sistema gamificado como son también conocidos, una interacción inmersiva e intuitiva que estimula de forma óptima los sentidos y sirve como motivador para la ejecución de esta dolorosa e incómoda actividad.

Sobre las reflexiones anteriores y como parte del impulso de nuevas estrategias, González (2017) en su proyecto de desarrollo de una suite de juegos serios, persiguió como objetivo principal diseñar una interfaz web contentiva de varios micro juegos de aventura para servir como herramienta en la rehabilitación de personas con movilidad reducida, a los fines, de que esta novedosa técnica sirviera para estimular de forma entretenida y motivadora dicho proceso. En esta interfaz web, se utilizó además como herramienta de hardware un dispositivo Kinect para la captura de movimiento.

Asimismo, esta estrategia de gamificar numerosos entornos de rehabilitación apunta a la promoción y consecución de objetivos orientados a la motivación, inclusión social y laboral de personas con movilidad reducida a través de metodologías optimizadas, muchas de ellas, inmersivas para estimula positivamente al progreso de las mismas, tal y como lo presenta González (2014) en su conferencia acerca de "gamificación aplicada a la educación y salud", donde desnuda una seria de técnicas y estrategias de gamificación aplicadas en el área de la salud muy específicamente en la rehabilitación física.

Por consiguiente, con el perfeccionamiento de este novedoso conjunto de técnicas que se apalancan en la gamificación como elemento optimizador de procesos de rehabilitación a través de terapias físicas, haciendo uso de software especializado, muchos de ellos con RV, RA y dispositivos como Kinect y gafas 3D, se ha logrado generar entornos inmersivos y controlados tal como lo define Márquez, Martinez, \& Rolón (2011) los cuales han impactado de forma efectiva en la calidad de vida de este grupo vulnerable debido a su movilidad reducida.

Este nuevo quehacer permite delinear una nueva cultura social más consciente e inclusiva al tiempo de recrear nuevas estrategias que permitan ser socialmente responsables desde nuestro entorno como individuos y poder establecer líneas claras de acción sobre estos grupos que hasta hace pocos años y en la actualidad sufren de algún tipo de discriminación o segregación.

Como se puede inferir, para estos investigadores la gamificación ha mostrado resultados positivos, en una proporción que ha permitido observar mejoras sustanciales en pacientes con movilidad reducida posterior al haberse expuesto a este tipo de terapias.

Para finalizar, se desarrolla el presente estudio de tipo documental con la firme intención de analizar los avances que presenta la gamificación como estrategia para optimizar los procesos de rehabilitación en personas con discapacidad, a los fines, de desarrollar una suite 
de interfaces 3D para una gama reducida de dispositivos móviles y de escritorio para esta condición, por lo cual, este producto está relacionado con el proyecto denominado desarrollo de suite interactiva con fines de rehabilitación para personas con movilidad reducida.

En el contenido de la presente investigación se analizó a detalle en primer lugar el GAMING, una filosofía estratégica para casos complejos; donde se abordó la temática acerca de la gamificación de entornos a través de videojuegos ó juegos serios como también son conocidos, incluyendo además, la adición de accesorios tales como Kinect, gafas 3D, cámaras, entre otros equipos para desarrollar espacios más inmersivos e intuitivos en ciencias tan complejas como la medicina, con especial atención, en la rehabilitación de discapacidades.

Como punto número dos se desarrolló la rehabilitación y GAMING, donde se exploró algunas de las causas por las cuales se hace necesaria la rehabilitación de pacientes, como también, las alternativas que presentan una serie de investigaciones en el tema, punto donde señalan, las terapias con GAMING para ciertas discapacidades.

El punto número tres, Entornos de Desarrollo para el GAMING en personas con discapacidad; en este apartado se reconocen y explican las herramientas más utilizadas para el desarrollo de aplicaciones en 2D y 3D y la integración de accesorios en sistemas gamificados para atender a procesos de rehabilitación en discapacitados.

\section{Fundamentación teórica}

\section{GAMING, una filosofía estratégica para casos complejos}

De acuerdo a los postulados de Teixes (2015), el GAMING por su nombre en inglés, es una estrategia innovadora que toma mayor vigencia con el paso del tiempo al incluir entornos desarrollados en 3D, Realidad Virtual ó Realidad Aumentada para propicios espacios más inmersivos y sensoriales que junto con accesorios como Kinect y gafas 3D proporcionan mayor interactividad con el usuario, lo cual lo hace aún más motivador. En este sentido, estas distinguidas áreas interactivas por sus propiedades inclusivas se traspolan en la actualidad a un sinnúmero de espacios científicos tomando presencia en ambientes como la salud, empresa, gobierno y educación, por lo cual, el estudio de los entornos virtuales como el GAMING en panoramas no lúdicos influye positivamente en el comportamiento del individuo al estimular y motivar las acciones dentro de cada contexto lo que establece un parámetro vital y una tendencia notoria hacia la implementación de esta estrategia renovadora en estos escenarios, muy especialmente, en la salud con fines de rehabilitación de discapacidades.

A tales efectos y en concordancia con Madina Alimanova, Nurgul Kurmangaiyeva, Dinara Kozhamzharova, Gulnar Tyulepberdinova, \& Aray Kassenkhan (2017) quienes en su investigación titulada "Gamification of Hand Rehabilitation Process Using Virtual Reality Tools: Using Leap Motion for Hand Rehabilitation" definen la gamificación a través del concepto de la realidad virtual, como una herramienta que posibilita grandes oportunidades para desplegar nuevos técnicas en aras de perfeccionar la fisioterapia tradicional. En esta investigación se hace uso de la herramienta Leap Motion, que ha tenido gran acogida en los últimos años por sus amplias aplicaciones, por lo cual, al comparar estas evidencias, es 
menester para la presente investigación reseñar las ventajas del GAMING como estrategia para la rehabilitación de personas con discapacidad porque abre una ventana creativa, innovadora, inmersiva e inclusiva en los espacios de la fisioterapia.

Por su parte Lamyae, Ali, \& Fernández (2017), resume el conocimiento actual de la gamificación en los espacios del e-Health o cibersalud, término acuñado por la Organización Mundial de la Salud y cuáles son los aportes de esta técnica y la del juego serio en contextos de salud y bienestar relacionados específicamente con la rehabilitación de enfermedades crónicas, estudio que permite definir a la rehabilitación de discapacidades motoras ya como una filosofía aterrizada en estos espacios que hasta hace unos años solo era definida como una herramienta exploratoria.

En este mismo contexto, Janssen, y otros (2017) le da una nueva dinámica a la gamificación definiendo que la misma está determinada por particularidades como el atractivo, la motivación y el compromiso las cuales son variables significativas en las mediaciones de terapia física, ya que implican sistemas dopaminérgicos concernientes con la recompensa en el cerebro que son acreditados por proporcionar el aprendizaje a través de la potenciación a largo plazo de las conexiones neuronales, postulados que le dan a la presente investigación un enfoque claro concerniente al grado estratégico del uso correcto de esta serie de procedimientos tecnológicos y como han permeado hasta convertirse en una filosofía clara de innovación en escenarios complejos como lo es la rehabilitación de múltiples discapacidades.

Por su parte, Pérez \& Bautista (2017) diseño en su investigación un sistema que puede medir parte de la goniometría de la mano y utilizar dicha información para establecer a través de una interfaz gráfica un juego serio y donde la investigación de Peláez (2018) logró con el uso de la gamificación en su primera etapa la rehabilitación de personas con movilidad reducida, evidenciando que esta sistematización se logra en virtud de que el método tradicional es doloroso, pesado y produce desmotivación, lo cual es contrarrestado con la gamificación para que el paciente se entretenga y sienta motivado y no centrado en el hecho de tener la discapacidad, investigaciones que apuntalan una filosofía firme y una estrategia clara a la hora de establecer herramientas novedosas en referidas a contrarrestar las discapacidades motoras.

En este orden de ideas y para finalizar, Casanova (2016) en su investigación evalúa los efectos que produce la esclerosis múltiple desde la perspectiva de las discapacidades motoras, sensoriales, cognitivas y comportamentales. Para ello, resalta la importancia de los ejercicios físicos terapéuticos a través de un sistema gamificado para contrarrestar los efectos de esta terrible enfermedad, demostrando los beneficios que estas novedosas estrategias de automatización de métodos convencionales, pueden lograr con personas con movilidad reducida.

De las reflexiones anteriores y para finalizar se puede inferir que el GAMING como estrategia per sé ha permeado muchas ciencias complejas en la búsqueda de la sistematización de los métodos tradicionales, cuestión que ha hecho posible que los entornos de hoy día sean visto de forma más inclusiva, inmersiva y dinámica que en tiempos anteriores, por esta razón, la gamificación se ha convertido en una filosofía potenciadora en los espacios donde presenta mejores opciones que los métodos habituales. 
A tales efectos, se hace indispensable considerar esta nueva filosofía de sistematización de espacios donde la experimentación sensorial juega un papel importante para el desarrollo de tareas complejas, en este caso particular, la rehabilitación de discapacidades, pues ofrece un efecto motivacional e inclusivo superior a los métodos cuotidianos.

\section{Rehabilitación y GAMING}

Según Carmona, González \& Garcia (2017) definen que en la actualidad el accidente cerebrovascular presume una de las causas más significativas de mortalidad alrededor del mundo, en este sentido y en concordancia con estadísticas de la Organización Mundial de la Salud 17.5 millones de personas murieron por enfermedades cardiovasculares en el año 2012, de ellas, 6.7 millones fueron producto de accidentes cerebrovasculares, estadísticas que contextualizan la clara emergencia de consolidar estrategias de rehabilitación para revertir los efectos de movilidad reducida en pacientes que han sufrido este tipo de accidentes.

A este respecto, Velarde (2015) en su investigación discernió que en los últimos diez años se han gestado un gran cumulo de exploraciones en procesos de rehabilitación con el apoyo de nuevas tecnologías, tal es el caso de Gonzalez (2014), Ferrarini, González, Zapata, Olguín, \& Ormeño (2016), Gonzalez (2017) y Peñeñory, Bacca, $\&$ Cano (2018). No obstante, existen investigaciones que hacen uso de accesorios tecnológicos como Kinect que fungen como agentes de captura de movimiento en estos escenarios inmersivos entre ellos Hernández, y otros (2018), Cano, Collado, \& Canode-la-Cuerda (2017) y Pinilla (2017) los cuales han traído resultados efectivos para pacientes con discapacidad. A tales efectos, el desarrollo de estas tecnologías inclinadas hacia la gamificación permite que se pueda capturar incluso información concerniente al movimiento del cuerpo humado.

En este orden de ideas según la percepción de Mulero (2017), enfatiza el tratamiento temprano en pacientes con enfermedades cerebrovasculares y luego de una revisión sobre investigaciones acerca de terapias de neurorehabilitación, concluye en el despliegue de sistemas de realidad virtual para optimizar este tipo de terapias. En este apartado, se diseñan dos videojuegos musicales a los fines de ser implementados sobre este tipo de terapias.

Asimismo, Acuario (2017) plantea en su investigación un sistema inclusivo enfocado en la rehabilitación motora fina para pacientes adultos con daño cerebral adquirido, en un primer término, sin embargo puede ser utilizado en niños, jóvenes y para cualquier otra patología. Este software como lo explica el investigador tiene dos grado de inmersión: sonoro estimulación de fuerza a través del dispositivo háptico y finalmente para validar la herramienta utilizo el test de usabilidad SUS sobre un grupo de pacientes con Daño Cerebral Adquirido de nivel leve y moderado.

Desde la revisión de la evidencia científica se puede constatar los esfuerzos de muchas investigaciones de aplicar novedosas herramientas tecnológicas dentro de la rehabilitación, todas relacionadas, con la gamificación de escenarios diversos para muchos tipos de discapacidades, así lo demuestran Mantilla \& Martinez (2017) y Suriá (2018) en sus investigaciones, donde infieren que en la última década se han venido desarrollando innovadores avances científicos con respecto a la fisioterapia y en la creación de dispositivos 
y sistemas tecnológicos utilizados en la intervención de pacientes con diversos tipos de patologías. A todas luces, el uso de sistemas gamificados han venido innovando muchos espacios dentro de los procesos de rehabilitación con múltiples herramientas tanto en aplicaciones en 2D como en sistemas inmersivos donde se ubican los sistemas gamificados en $3 \mathrm{D}$, adjuntando accesorios que permiten capturar movimientos como lo es el Kineck y múltiples cámaras para provocar el impulso de estas fisioterapias.

Resulta asimismo interesante, la investigación de Muñoz \& Calvo (2017) donde se analiza y figuran los axiomas científicos sobre los efectos de la aplicación de la terapia de Realidad Virtual en el miembro superior en pacientes con ictus crónico. Dentro de sus conclusiones esgrime que la terapia de realidad virtual en combinación con la terapia convencional traduce en mejoras adicionales con respecto al uso exclusivo de la terapia convencional. Los estudios practicados muestran beneficios en la movilidad, la fuerza, la calidad y la cantidad de movimiento del miembro superior.

Díaz Ayala \& Rodriguez (2016) en su investigación titulada "Prescripción de los programas de realidad virtual aplicados a pacientes con lesión motora de origen neurológico: una revisión sistemática." plantea identificar desde el ámbito científico la prescripción utilizada en los programas de fisioterapia con realidad virtual en pacientes con lesión motora de origen neurológico. En su investigación concluye la certeza de establecer aplicaciones de realidad virtual en los programas de fisioterapia en pacientes con lesión motora de origen neurológico.

Finalmente, en su investigación Cartes (2017) evalúa los dispositivos de realidad virtual para la evaluación y mejoramiento del balance postular en adultos mayores. En el estudio, el investigador determina que la realidad virtual puede ser eficazmente implementada como un método de diagnóstico, entrenamiento y rehabilitación de adultos mayores. Asimismo, en su investigación determina que las mejoras logradas mediante el entrenamiento virtual con la consola Wii son significativas y similares a las obtenidas mediante ejercicios convencionales, de igual forma, la implementación del entrenamiento virtual incrementa la adherencia al tratamiento.

Luego de realizar el recorrido por los estudios de los autores antes mencionados se infiere que los procesos tradicionales de rehabilitación resultan dolorosos y en lugares que presumen poca motivación, asimismo, alejados o poco accesibles por personas con discapacidad motora, hecho por el cual, la rehabilitación con sistemas gamificados presentan mejoras considerables con respecto al método tradicional, pues permite servir de estímulo sensorial y motivacional por tanto están desarrollados en ambientes más dinámicos e inmersivos, muchas veces en $3 \mathrm{D}$, RV o RA, que adicionan accesorios que sirven de interfaces para capturar los movimientos de la persona quien ejecuta el proceso de fisioterapia y como estrategia inclusiva para personas con impedimentos motrices que no pueden desplazarse hacia los lugares tradicionalmente dispuestos para estas fisioterapias.

\section{Entornos de Desarrollo para el GAMING en personas con discapacidad}

Hoy en día existen una diversa variedad de lenguajes de programación para gamificar escenarios, también conocidos como entornos de desarrollo, los cuales a través de un abanico incontable de librerías pueden trasladar escenarios de cualquier índole, incluidos 
los escenarios inmersivos muy en boga para el diseño de entornos 3D. Estos entornos de desarrollo pueden generar aplicaciones de índole general, desde aplicaciones gamificadas para el sector automotriz, como aplicaciones más sensibles como las dispuestas para el sector salud, en este caso, lo referente a la rehabilitación.

En tal respecto, diversas investigaciones se han dado lugar para implementar ciclos de rehabilitación asistida con tecnología gamificada que han dado exitosos pasos en adelanto científico y han permitido reingeniar e innovar en este terreno a veces complejo.

En su proyecto de investigación Pelaez (2018) diseño un videojuego terapéutico para personas con movilidad reducida haciendo uso de Unity 3D, un motor de videojuegos multiplataforma que permite crear entornos inmersivos con gran calidad y resolución que permite diseñar juegos en 3D. Adicional a este motor multiplataforma empleo el lenguaje de programación $\mathrm{CA}$, un lenguaje de programación orientado a objeto de Microsoft perfectamente compatible con el motor Unity 3D. La unión de ambas aplicaciones permitió crear efectivamente una interfaz amigable para el desarrollo de terapias para personas con enfermedades neuromusculares.

Por otra parte, Sandoval, Carreño, Estrada, \& Leyva (2016) en su investigación denominada "Development of a videogame to improve communication in children with autism" implemento para su sistema gamificado, en este caso denominado videojuego, el motor Unity 3D en su versión gratuita compatible con la plataforma Windows, Androis y iOS (Sistema operativo para iPhone), asimismo, hizo uso del software GIMP (GNU Image Manipulation Program).

El videojuego se implementó utilizando el motor de videojuegos Unity 3D, el cual cuenta con una versión estándar de desarrollo gratuita que permite publicar en las plataformas Windows, Android y iOS, así como la posibilidad de subir el juego a una página web mediante Unity Web Player. La edición de las imágenes se llevó a cabo con el software GIMP (GNU Image Manipulation Program). La primera versión del videojuego se ejecuta en móviles Android sin considerar la conexión a Internet. En esta investigación se planteó la implementación de un videojuego para atender a discapacidades de tipo cognitiva para lo cual se construyó un prototipo de juego serio tomando como base acciones diseñadas por especialistas en la materia de terapias para niños autistas.

Otras investigaciones como es el caso de Pinilla (2017) diseño un juego serio para fisioterapias de rehabilitación motriz y cognitiva dispuestas con realidad virtual. En su desarrollo hizo uso del motor de videojuegos Unity 3D, coincidiendo con las herramientas de los autores ante citados en virtud de que permite el desarrollo inmersivo al tiempo de ofrecer características multiplataformas. En su estudio, coincide que el diseño de software para gamificar debe considerar los siguientes resultados:

- Lograr la mayor utilidad gráfica del terminal donde se va a ejecutar la aplicación de videojuego, y de sus propiedades para reproducir gráficos en alta resolución y ambientes inmersivos.

- Una utilidad que permita desarrollar una suite de aplicaciones completa.

- Que esta herramienta de desarrollo tenga la característica de ser multiplataforma, en este sentido, computadoras, dispositivos móviles, ambientes web, entre otros. 


\section{Metodología}

Metodológicamente la investigación se desarrolló bajo el paradigma cualitativo el cual trata de identificar la naturaleza profunda de las realidades, su estructura dinámica, aquella que da razón plena de su comportamiento y manifestaciones (Martínez, 2012). En cuanto al conocimiento científico surgido como producto de este estudio corresponde a la afirmación positiva de las teorías del GAMING y la rehabilitación, a través de la aplicación del método científico, es por ello que el conocimiento generado busca explicar causalmente los fenómenos indagados por medio de las teorías ya mencionadas, y por tanto se concibe a la razón como medio para otros fines; es decir, una razón instrumental. (Perez, 2010).

Por otro lado, busca establecer las relaciones o conexiones existentes entre los fenómenos observados, por lo que igualmente se establece que la observación a la que se incurre es preminentemente objetiva, independiente del sujeto que conoce. El investigador permanece neutral ante la realidad estudiada, con el fin de establecer las relaciones constantes que existen en el fenómeno estudiado, permitiendo prever racionalmente su comportamiento futuro. (Ortecho, 2007).

En tal sentido, el propósito de esta investigación, se concentra en analizar el GAMING desde la perspectiva de un conjunto sistemático de técnicas de desarrollo de aplicaciones y accesorios tecnológicos orientados hacia los videojuegos, como una estrategia para la rehabilitación de personas con discapacidad, recurriendo a la obtención de información mediante el uso del método científico para el cumplimiento lógico del objetivo propuesto.

Finalmente, la presente investigación se llevó a cabo bajo un estudio bibliográfico con un diseño documental, transaccional empleando las categorías GAMING y rehabilitación para proyectar una matriz de análisis desde donde poder establecer las relaciones de los tópicos de acuerdo a diferentes autores y en este sentido poder enmarcar de forma teórica la justificación del uso de los sistemas gamificados como novedosas herramienta para la rehabilitación de personas con diversas discapacidades motrices (Tamayo y Tamayo, 2006).

\section{Resultados}

En la última década, se han hecho evidente los esfuerzos para el desarrollo de nuevas tecnologías en múltiples entornos y más aún en las ciencias aplicadas, lo que ha permeado considerablemente el escenario de la medicina, motivo de esta investigación. Estos adelantos per sé han sido apropiados por diversos tipos de usuario, considerando a cada tipo de usuario en un sentido amplio como aquel que selecciona la tecnología que le favorece en su entorno particular y le permite crecer desde su interior para completar su actividad diaria.

Sobre la base de las ideas expuestas y enfocados en la temática de la discapacidad, los avances tecnológicos han desplegado e incorporado una importante cultura hacia lo referente a la automatización y sistematización, en algún sentido, de los métodos tradicionales admitiendo el acceso y habilidad de desarrollar procesos fisioterapéuticos de forma más cómoda, motivadora e inmersiva que resulte atractiva para el paciente que hasta hace poco tiempo atrás debía lidiar con largos ciclos de terapias incomodas, desplazamiento social, laboral y estigmatización por parte de esta cultura de perfección. 
Se indica asimismo, que la gamificación ha marcado un parámetro primordial en el perfeccionamiento de nuevos escenarios inmersivos, entornos dinámicos que ofrecen particularidades únicas a la hora de desplegar atmósferas que desplazan las formas tradicionales y capturan la atención de usuario por el uso de la virtualización, imágenes 3D, RV o RA, por lo cual, el siguiente cuadro muestra las últimas tecnologías relacionadas con el desarrollo de ambientes gamificados para discapacidades haciendo énfasis en herramientas para programar dichos sistemas.

Tabla 1. Entornos de programación para sistemas gamificados

\begin{tabular}{cc}
\hline Entornos de programación & Función \\
\hline Unity 3D & $\begin{array}{c}\text { Es un motor de videojuegos multiplataforma que está disponible } \\
\text { para diferentes sistemas operativos como Microsoft, Linux y } \\
\text { OS X. Permite compilar aplicaciones en diversas plataformas } \\
\text { objetivos tales como Web, Pc, Dispositivos móviles, Smart TV, } \\
\text { consolas y dispositivos de realidad extendida }\end{array}$ \\
\hline Blender & $\begin{array}{c}\text { Es un sistema informático multiplataforma para modelado, } \\
\text { renderización, creación y animación de gráficos en 3 } \\
\text { dimensiones. Este software oofrece compatibilidad con } \\
\text { Windows, Mac OS, Linux, Android, entre otros. }\end{array}$ \\
\hline C\# & $\begin{array}{c}\text { Es un lenguaje de programación orientado a objetos creado por } \\
\text { Microsoft que se encuentra en su plataforma .NET. Forma parte } \\
\text { del estándar ISO/IEC 23270 }\end{array}$ \\
\hline JavaScript & $\begin{array}{c}\text { Es un lenguaje orientado a objetos, utilizado como interfaz } \\
\text { client-side (lado del cliente) que se implementa en navegadores } \\
\text { web para optimizar la vista de usuario en plataformas web. Es un } \\
\text { software de desarrollo libre. }\end{array}$ \\
\hline \multirow{2}{*}{ Phyton } & $\begin{array}{c}\text { Es un lenguaje de programación multiplataforma y multiparadigma, } \\
\text { pues, soporta orientación a objetos, desarrollo imperativo y } \\
\text { desarrollo funcional. Es un software de desarrollo libre. }\end{array}$ \\
\hline
\end{tabular}

Fuente: Elaboración propia (2019).

Asimismo, los sistemas gamificados hacen uso de accesorios que permiten interacciones más dinámicas, por lo cual, se presenta a continuación una tabla con los más utilizados en la actualidad. 
Tabla 2. Accesorios utilizados en sistemas gamificados para discapacidad

\begin{tabular}{cc}
\hline Dispositivo & Función \\
\hline Microsoft Kinect & $\begin{array}{c}\text { Dispositivo creado por Microsoft para ser utilizado como controlador } \\
\text { que permite al usuario interactuar con una consola de video juego sin } \\
\text { tener contacto físico con ella, este reconoce gestos, objetos, imágenes y } \\
\text { comandos de voz. }\end{array}$ \\
\hline Gafas 3D Móvil y Gafas & $\begin{array}{c}\text { Son paneles LSD que proyectan imágenes en cada ojo para recrear } \\
\text { 3D para PC }\end{array}$ \\
& $\begin{array}{c}\text { en algunas imágenes bidimensionales. Ellas por su parte, tienen } \\
\text { diferentes diseños que soportan interfaces tanto móviles como para } \\
\text { computadoras, estas últimas, tienen dos controladores manuales que } \\
\text { reconocen la posición del usuario. }\end{array}$ \\
\hline Leap Motion & $\begin{array}{c}\text { Es un dispositivo periférico USB de captura de movimiento. Hace uso } \\
\text { de dos cámaras infrarrojas monocromáticas y 3 LED para capturar } \\
\text { movimiento a una distancia no superior de un metro. Este dispositivo es } \\
\text { utilizado para la captura manual en 3D. }\end{array}$ \\
\hline
\end{tabular}

Fuente: Elaboración propia (2019).

En la actualidad la mayoría de los sistemas gamificados están diseñados para contar con escenarios inmersivos e interactivos, es decir, diseñados en 3 dimensiones para poder captar aún más la atención de aquellos usuarios que lo necesiten, en este sentido, existen en el mercado numerosos lenguajes de programación para el diseño de videojuegos, algunos son de distribución gratuita y otros no, sin embargo, la elección a la hora de desarrollar con alguno de ellos queda a la elección del programador en virtud de que estas herramientas presentan más o menos soporte a la hora de desarrollar alguna aplicación en particular.

Adicional a esta característica, se suelen utilizar accesorios que estimulen aún más la experiencia virtual como es el caso del Microsoft Kinect que eventualmente permite al jugador poder interactuar con el sistema gamificado sin necesidad de utilizar algún tipo de control o mando.

\section{Conclusiones}

Los sistemas gamificados permiten descubrir nuevos escenarios más creativos, inmersivos e interesantes que sirven de estímulo para personas que presentan diversas discapacidades, considerando, que una de las causas más frecuentes de la deserción en los ciclos fisioterapéuticos corresponde a lo dolorosa, incomoda y rígida de las mismas.

Es de hacer notar, que en el mercado y desde la investigación existen numerosos estudios que han demostrado el uso efectivo de las terapias con sistemas gamificados que han dado excelentes resultados en la mejoría de pacientes con discapacidades multiples, lo que sugiere que este tipo de terapias en combinación con los métodos tradicionales son un mecanismo efectivo para tratar este tipo de enfermedades.

Finalmente, ante estos novedosos escenarios inmersivos y creativos que se apalancan en la tecnología estamos frente a una nueva generación de instrumentos que permiten generar 
mayor motivación a pacientes con discapacidades motoras para de esta forma poder ser integrado a la sociedad y al ambiente laboral generando motivación desde estos ambientes tecnológicos.

\section{Referencias bibliográficas}

Acuario, A. (2017). Implementación de una herramienta virtual mediante un dispositivo háptico para la rehabilitación de pacientes con daño cerebral adquirido (Tesis de pregrado). Latacunga, Ecuador.

Alfonso, J., \& Martinez, J. (2017). Innovación y tecnología en fisioterapia: Futuras herramientas de intervención. Movimiento Científico, 37-43.

Cano, M., Collado, S., \& Cano, R. (2017). Videojuegos comerciales en la rehabilitación de pacientes con ictus subagudo: estudio piloto. Revista de Nerología, 337-347.

Carmona, A., González, R., \& Garcia, I. (2017). Tratamiento rehabilitador en pacientes ancianos post-ictus. Revisión sistemática. Revista Mexicana de Medicina Física y Rehabilitación, 51-56. Obtenido de medigraphic.org.mx.

Cartes, J. (2017). Dispositivos de realidad virtual para la evaluación y mejoramiento del balance postular en adultos mayores. Estado del arte. IJMSS International. Journal of medical and surgical science, 1266-1273.

Casanova, M. (2016). Validación de un exergame para esclerosis múltiple a través de análisis biomecánico usando teoría de sistemas dinámicos. Pereira, Colombia.

Díaz, L., \& Rodriguez, J. (2016). Prescripción de los programas de realidad virtual aplicados a pacientes con lesión motora de origen neurológico: una revisión sistemática. fisioGlía, 45-54.

Ferrarini, C., González, M., Zapata, S., Olguín, L., \& Ormeño, E. (2016). http://sedici. unlp.edu.ar. Recuperado el 28 de 05 de 2019, de http://sedici.unlp.edu.ar/ handle/10915/54096

Gonzaléz, C. (2014). Estrategias Gamificación aplicadas a la Educación y a la Salud. San Cristóbal de La Laguna, Tenerife, España.

Gonzaléz, C. (2017). Diseño e implementación del entorno de videojuego serio de rehabilitación del proyecto "Blendex" (Tesis de pregrado). Universidad Politécnica de Madrid: Escuela Técnica Superior de Ingeniería y Sistemas de Telecomunicación, Madrid, España.

Hernández, J., Rauch, M., Rivas, D., Asenio, P., Asenio, C., \& Solis, M. (2018). Efectos del entrenamiento con Xbox Kinect sobre la movilidad funcional en adultos mayores. Una revisión breve. Revista Ciencias de la Actividad Física, 1-10. doi:https://doi.org/10.29035/rcaf.19.2.2

Janssen, J., Verschuren, O., Renger, W., Ermers, J., Ketelaar, M., \& van Ee, R. (2017). Gamification in Physical Therapy: More Than Using Games. Pediatric Physical Therapy, 29(1):95-99. 
Lamyae, S., Ali, I., \& Fernández, J. (2017). A systematic review of gamification in e-Health. Journal of Biomedical Informatics, 32.

Madina, S., Nurgul, D., Kozhamzharova, D., Gulnar, G., \& Kassenkhan, A. (2017). Gamification of Hand Rehabilitation Process Using Virtual Reality Tools Using Leap Motion for hand rehabilitation. IEEE, 336.

Márquez, R., Martinez, Y., \& Rolón, O. (2011). Impacto del Programa de Terapia de Realidad Virtual. Rev, 12(1): 16-26.

Martínez, M., (2012). Nuevos fundamentos en la investigación científica. México: Trillas

Mulero S. (2017). Rehabilitación motora con instrumentos musicales en entorno virtual (Tesis de pregrado). Valladolid.

Muñoz, R., \& Calvo, I. (2017). Efectos de la terapia de realidad virtual en el miembro superior en pacientes con ictus: Revisión sistemática. Sociedad española de rehabilitación y medicina física, 1-10.

Ortecho. (2007). Ciencia y tecnologìa para el hábitat popular. Editorial Granice.

Paz, M., Perugachi, E., Romero, D., Sappa, A., \& Vintimilla, B. (2015). Análisis del movimiento de las extremidades superiores aplicado a la rehabilitación física de una persona usando técnicas de visión artificial. Revista Tecnológica ESPOL - RTE, 1-7.

Peláez, J. (2018). "Blexter" - Desarrollo de videojuego terapéutico para personas con movilidad reducida. Universidad Politécnica de Madrid: Escuela Técnica Superior de Ingeniería y Sistemas de Telecomunicación, Madrid, España.

Peñeñory, V., Bacca, A., \& Cano, S. (2018). Propuesta metodológica para el diseño de juegos serios para la rehabilitación psicomotriz de niños con discapacidad auditiva. Revista Campus Virtuales, 47-54.

Pérez, J. (2010). Enfoques metodológicos. Editorial La Salle.

Pérez, A., \& Bautista-Blanco, L. (14 de 08 de 2017). http://memorias.somib.org.mx. Recuperado el 29 de 05 de 2019, de http://memorias.somib.org.mx/index.php/ memorias/article/view/114

Pinilla, I. (2017). Juego serio para terapias de rehabilitación motora y cognitiva con realidad virtual (Tesis de pregrado). Valladolid.

Sandoval, J., Carreño, M., Estrada, I., \& Leyva, A. (2016). Development of a videogame to improve communication in children with autism. IEEE.

Suriá, R. (2018). Conducta prosocial de los estudiantes hacia la discapacidad y relación con la inteligencia emocional. Ediciones Universidad de Salamanca, 27-41.

Tamayo, M. (2006). El proceso de la investigación científica. México: Limusa.

Teixes, F. (29 de 06 de 2019). books.google.es. Obtenido de https://books.google.es/books?id= SipNCgAAQBAJ\&printsec $=$ frontcover $\& \mathrm{hl}=\mathrm{es} \# \mathrm{v}=$ onepage $\& \mathrm{q} \& \mathrm{f}=$ false 